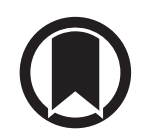

CrossMark

\section{Nasopharyngeal aspirate value in paediatric chronic lower respiratory tract illness}

To the Editor:

Respiratory viruses are a common cause of illness in children and are responsible for high morbidity and hospitalisation [1]. The role of infectious agents in chronic/recurrent lower respiratory tract illness (LRTI) is not clearly defined, whereas it is well known in acute forms and in exacerbation of respiratory diseases [2-4]. We have previously demonstrated a high frequency of viruses, particularly human rhinovirus (HRV), in children affected by chronic/recurrent LRTI whose bronchoalveolar lavage (BAL) showed changes in cellularity and inflammatory cytokines [5]. BAL is the gold standard for sensitive detection of infective agents of the lower respiratory tract, even if invasive. Nasopharyngeal aspirate (NPA), nasopharyngeal swab and nasal wash have been investigated to find an easier and more reliable technique for viral diagnosis but never in children affected by different forms of chronic/recurrent LRTI [6-8]. Our study aimed to investigate the presence of viral genomes testing the value of NPA in comparison with BAL in a large paediatric population affected by different forms of chronic/recurrent LRTI.

We studied 133 children (mean \pm SD age: 77.6 \pm 40.2 months, 68 females and 65 males), consecutively admitted to our centre from March 2008 to March 2012 for chronic/recurrent LRTI (figure 1). A clinical definition of these disorders has been made by expert paediatric pulmonologists as previously reported [5] and all the diagnoses are reported in figure 1. Exclusion criteria included: a recent episode of exacerbation, considered as symptoms of an upper respiratory disease and/or worsening of pulmonary function tests during the 2-week check-up before the visit/bronchoscopy; patients with cystic fibrosis, primary ciliary dyskinesia, primary and secondary immunodeficiency, tuberculosis and bronchial malformations. All patients underwent bronchoscopy with BAL and NPA. Written consent was obtained from parents of all participating children. Our Institutional Ethics Committee approved the study.

Reverse transcriptase (RT)-polymerase chain reaction (PCR), PCR or nested PCR of DNA/RNA extracted from all samples were used to detect the following respiratory viral genomes: HRV, adenovirus (AV), cytomegalovirus (CMV), Epstein-Barr virus (EBV), influenza virus A and B, metapneumovirus (MPV), herpesvirus (HHV)-6, HHV-7, HHV-8, parvovirus B19, parainfluenzavirus 1 and 3 and respiratory syncytial virus (RSV). Concordance between NPA and BAL was considered when at least one viral genome was present in both tools. All viral amplicons were sequenced as previously described [9] and phylogenetic analysis with MEGA7 software (Version 7.0.14; Biodesign Institute, Tempe, AZ, USA) was used to establish if the most frequently detected viruses were exactly the same genotypes in BAL and NPA. For statistical analyses, the proportion of concordant pairs and discordant pairs was computed and the agreement of detection between the two specimens was verified by the McNemar test for paired samples. To quantify the agreement between the two specimens for each virus, the $\kappa$ coefficient (95\% CI) was computed. To evaluate the reliability of NPA in the detection of viruses, sensitivity, specificity, positive predictive values (PPV) and negative predictive values (NPV) were also performed for each virus assuming BAL as the gold standard.

In 76 (57\%) out of 133 BALs, at least one viral genome was detected; in 32 (46\%) out of 76, coinfections were found.

@ERSpublications

Nasopharyngeal aspirate as a sensitive and specific surrogate tool for the detection of respiratory viruses in different forms of chronic/recurrent lower respiratory tract illness http://ow.ly/cd8S30jcwyu

Cite this article as: Lunardi F, Vuljan SE, Perissinotto E, et al. Nasopharyngeal aspirate value in paediatric chronic lower respiratory tract illness. Eur Respir J 2018; 51: 1701783 [https://doi.org/10.1183/ 13993003.01783-2017]. 


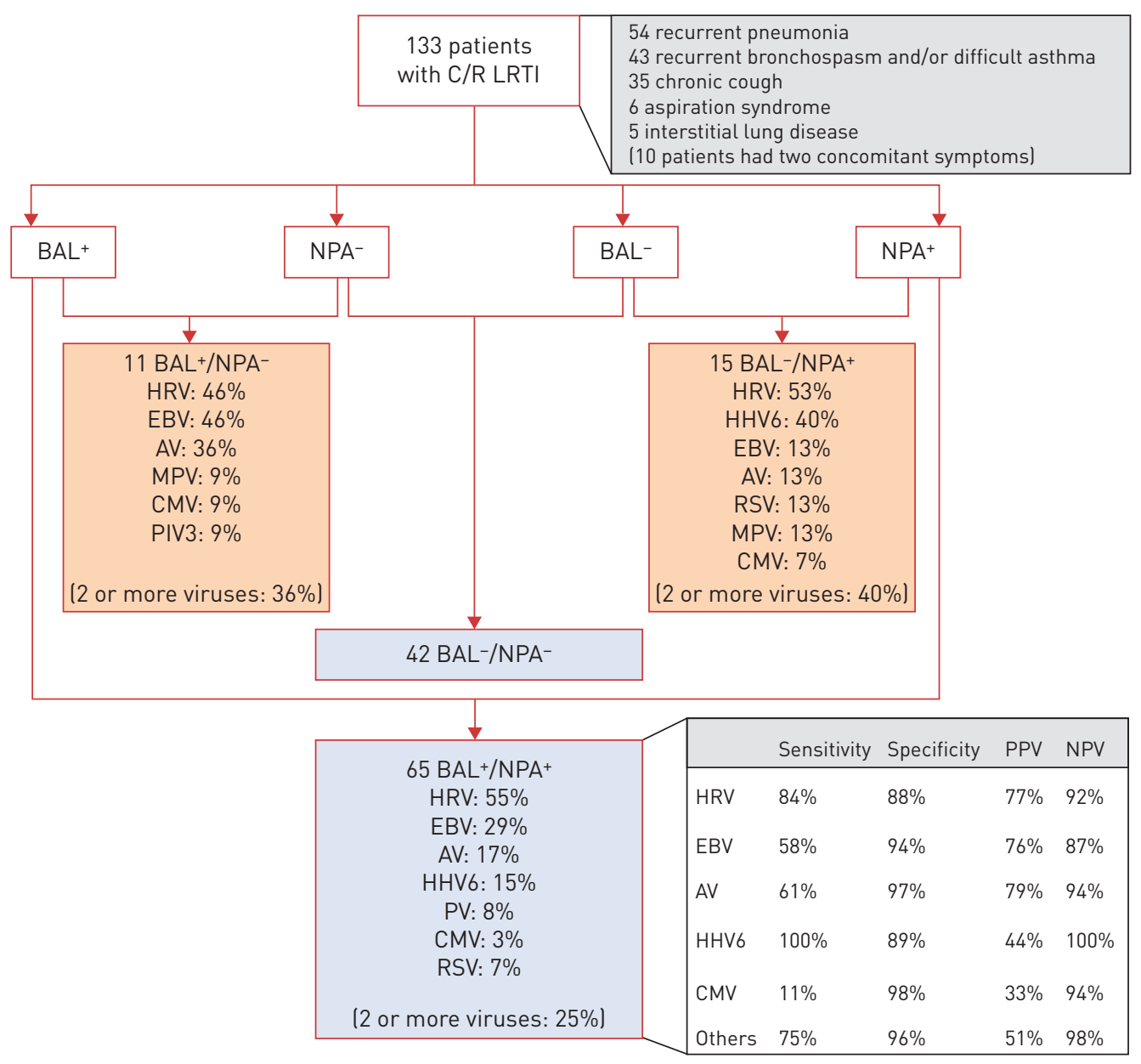

FIGURE 1 Data on concordance or discordance between the two tools and sensitivity/specificity of nasopharyngeal aspirate (NPA) by virus species. C/R LRTI: chronic/recurrent lower respiratory tract illness; BAL: bronchoalveolar lavage; PPV: positive predictive value; NPV: negative predictive value; HRV: human rhinovirus; EBV: Epstein-Barr virus; AV: adenovirus; HHV6: herpesvirus 6; CMV: cytomegalovirus; MPV: metapneumovirus; PV: parvovirus; PIV: parainfluenzavirus; RSV: respiratory syncytial virus; others include PV, MPV, PIV and RSV.

In $80(60 \%)$ out of 133 NPAs, at least one viral genome was detected; in 33 (40\%) out of 80, coinfections were found. The most frequent virus in both tools was HRV (figure 1). A viral concordance between BAL and NPA was detected in 107 (80.5\%) out of 133 patients (65 positive and 42 negative). When considering each type of virus, a good agreement was detected globally, mainly for more frequent viral genomes. In particular, the sensitivity of NPA in the investigation of HRV, the most frequently detected virus, was very high $(84 \%)$ as well as the specificity (88\%). Moreover, the PPV was $77 \%$ and the NPV was $92 \%$ (figure 1 ). Sensitivity of NPA in the detection of EBV and AV was lower (58 and 61\%, respectively) but specificity was always very high ( $94 \%$ and $97 \%$, respectively) as well as PPV (76\% and $79 \%)$ and NPV (87\% and $94 \%$, respectively) (figure 1). When considering low frequency-viruses (HHV6 and CMV), there was no concordance between the two tools.

Gene sequencing and phylogenetic analysis of HRV amplicons detected in BAL and NPA showed 100\% of sequence identity and very low genetic distance, thus confirming that the same virus was present in the upper and lower tract. In these patients, the detection of HRV in NPA can be considered a mirror of the lower airways with very good agreement between the two tools.

Many studies have been done to understand the role of HRV in the pathogenesis of respiratory diseases, from common colds to lower airway diseases. A few previous in vivo studies have demonstrated that persistence of HRV in the lower tract can lead to symptom persistence or severe progression of lung diseases. However, the majority of cases documented in the literature are related to patients with a significant degree of immunosuppression. Three individuals who had undergone lung transplantation, for instance, were reported as having persistence of HRV in the respiratory tract for over 12 months [10]. 
Patients with hypogammaglobulinaemia also appear to be prone to prolonged HRV shedding, with PCR positivity continuing for up to 55 days in a small group of such patients compared with 15 days in healthy individuals [11].

Our patients did not show any biohumoral signs of immunodeficiency and were not treated with systemic corticosteroids before bronchoscopy.

The presence of HRV in chronic/recurrent LRTI, as in our cases, could be responsible for the recurrence and persistence of symptoms and this aspect merits in depth investigation by mechanistic studies.

To the best of our knowledge, very few studies have investigated NPA as an alternative tool in the diagnostic approach to chronic/recurrent LRTI, but they differed from our research because the focus was on unpaired, non-contemporaneous NPA and BAL specimens [12] or, if paired, only in one type of chronic/recurrent LRTI [6]. A limitation of the study is the absence of a control group: the availability of healthy control cases in the paediatric field is limited because bronchoscopy with BAL is an invasive tool and can be done only when specific pathological conditions are present.

In conclusion, NPA is an excellent tool to exclude the presence of viral infections in the lower airways and it can represents a first level approach to exclude viral infections in children with chronic/recurrent LRTI.

Further investigations with the use of new molecular technologies (high throughput as next generation sequencing) could provide more precise characterisation of virus subtype and sequence variations from reference genomes.

Francesca Lunardi ${ }^{1}$, Stefania Edith Vuljan ${ }^{1}$, Egle Perissinotto ${ }^{1}$, Federica Pezzuto ${ }^{1}$, Sandro Malacrida ${ }^{2}$, Samuela Bugin ${ }^{3}$, Ilaria Bertozzi ${ }^{3}$, Deborah Snijders ${ }^{3}$, Stefania Rizzo ${ }^{1}$, Angelo Barbato ${ }^{3}$ and Fiorella Calabrese ${ }^{1}$

${ }^{1}$ Dept of Cardiac, Thoracic and Vascular Sciences, University of Padova, Padova, Italy. ${ }^{2}$ Dept of Biomedical Sciences, University of Padova, Padova, Italy. ${ }^{3}$ Dept of Paediatrics, University of Padova, Padova, Italy.

Correspondence: Fiorella Calabrese, Dept of Cardiac, Thoracic and Vascular Sciences, University of Padova, Via A. Gabelli 61, 35121 Padova, Italy. E-mail: fiorella.calabrese@unipd.it

Received: June 272017 | Accepted after revision: March 122018

Conflict of interest: None declared.

\section{References}

1 Bosis S, Esposito S, Niesters HG, et al. Role of respiratory pathogens in infants hospitalized for a first episode of wheezing and their impact on recurrences. Clin Microbiol Infect 2008; 14: 677-684.

2 Arden KE, McErlean P, Nissen MD, et al. Frequent detection of human rhinoviruses, paramyxoviruses, coronaviruses, and bocavirus during acute respiratory tract infections. J Med Virol 2006; 78: 1232-1240.

3 Kusel MMH, de Klerk NH, Holt PG, et al. Role of respiratory viruses in acute upper and lower respiratory tract illness in the first year of life. Pediatr Infect Dis J 2006; 25: 680-686.

4 Brodzinski H, Ruddy RM. Review of new and newly discovered respiratory tract viruses in children. Pediatr Emerg Care 2009; 25: 352-360.

5 Bugin S, Lunardi F, Bertuola F, et al. Pediatric chronic lower respiratory disorders: microbiological and immunological phenotype. Pediatr Pulmonol 2013; 48: 780-788.

6 Lambert SB, Whiley DM, O'Neill NT, et al. Comparing nose-throat swabs and nasopharyngeal aspirates collected from children with symptoms for respiratory virus identification using real-time polymerase chain reaction. Pediatrics 2008; 122: e615-e620.

7 Chan KH, Peiris JS, Lim W, et al. Comparison of nasopharyngeal flocked swabs and aspirates for rapid diagnosis of respiratory viruses in children. J Clin Virol 2008; 42: 65-69.

8 Wurzel DF, Marchant JM, Clark JE, et al. Respiratory virus detection in nasopharyngeal aspirate versus bronchoalveolar lavage is dependent on virus type in children with chronic respiratory symptoms. J Clin Virol 2013; 58: 683-688.

9 Mosser AG, Vrtis R, Burchell L, et al. Quantitative and qualitative analysis of rhinovirus infection in bronchial tissues. Am J Respir Crit Care Med 2005; 171: 645-651.

10 Kaiser L, Aubert JD, Pache JC, et al. Chronic rhinoviral infection in lung transplant recipients. Am J Respir Crit Care Med 2006; 174: 1392-1399.

11 Peltola V, Waris M, Kainulainen L, et al. Virus shedding after human rhinovirus infection in children, adults and patients with hypogammaglobulinaemia. Clin Microbiol Infect 2013; E322-E327.

12 Gerna G, Vitulo P, Rovida F, et al. Impact of human metapneumovirus and human cytomegalovirus versus other respiratory viruses on the lower respiratory tract infections of lung transplant recipients. J Med Virol 2006; 78: $408-416$. 